\title{
Validating BEM, Direct and Inverse Free Wake Models with the MEXICO Experiment
}

\author{
Daniel Micallef ${ }^{1,2}$, Menno Kloosterman ${ }^{1}$, Carlos Simão Ferreira ${ }^{1}$, Tonio Sant ${ }^{2}$, Gerard \\ van Bussel $^{1}$
}

December 13, 2009

\begin{abstract}
The primary objective of the MEXICO (Model Experiments in Controlled Conditions) project was to generate experimental data for validation of models for wind turbines. Kulite(c)pressure sensors were used for pressure measurements while Particle Image Velocimetry was used with the aim of tracking the tip vortex trajectory. The pressure measurements were carried out for both axial and yawed flow conditions with yaw angles of $15^{\circ}, 30^{\circ}$ and $45^{\circ}$. For the Particle Image Velocimetry measurements data was gathered for axial flow and for the $\pm 30^{\circ}$ yaw cases at a single tip speed ratio.

In this work, an inverse free wake lifting line model, a direct free wake model and a BEM model are validated with the MEXICO data. Particular emphasis is placed on the study of yawed flow conditions. The inverse free-wake model makes use of the experimental loads as input in order to find the distribution of inductions and angle of attack. The predictive capability of BEM may therefore be assessed based on this. Validation of the inverse free-wake model was performed by investigating the stagnation pressure prediction as well as the vortex trajectory prediction. This was done by means of the PIV data gathered from the MEXICO experiment. This PIV data was also used for validation purposes of the direct free-wake model. The differences in the angle of attack distributions in yawed flow with these models was studied in order to assess the difference in results between the use of 2D and 3D airfoil data.
\end{abstract}

\section{Introduction}

The MEXICO experiment was performed in the German-Dutch Wind Tunnels (DNW). Figure 1 shows the rotor model used for this experiment. The rotor has a diameter of $4.5 \mathrm{~m}$ and a hub diameter of $0.42 \mathrm{~m}$. The DNW wind tunnel has an open jet test cross section of $9.5 \times 9.5 \mathrm{~m}$. The measurements consisted of pressure distributions along the chord at five spanwise locations of the blade and Stereo Particle Image Velocimetry at certain locations of the flow field, including the tip vortex. The measurements included both yawed and axial conditions. The reader is referred to [1], [2] and [3] for more information regarding the setup, rotor configuration and measurements which were taken. Furthermore, experimental data validation along with some preliminary analysis has been done in [4].

In this work, the results from the MEXICO data are analyzed using blade element momentum (BEM), inverse and direct free-wake models. Particular attention for yawed

\footnotetext{
${ }^{1}$ DUWIND, TUDelft, Faculty of Aerospace Engineering, Kluyverweg 1, 2629 HS, Delft, The Netherlands

${ }^{2}$ University of Malta, Department of Mechanical Engineering, Malta.
} 


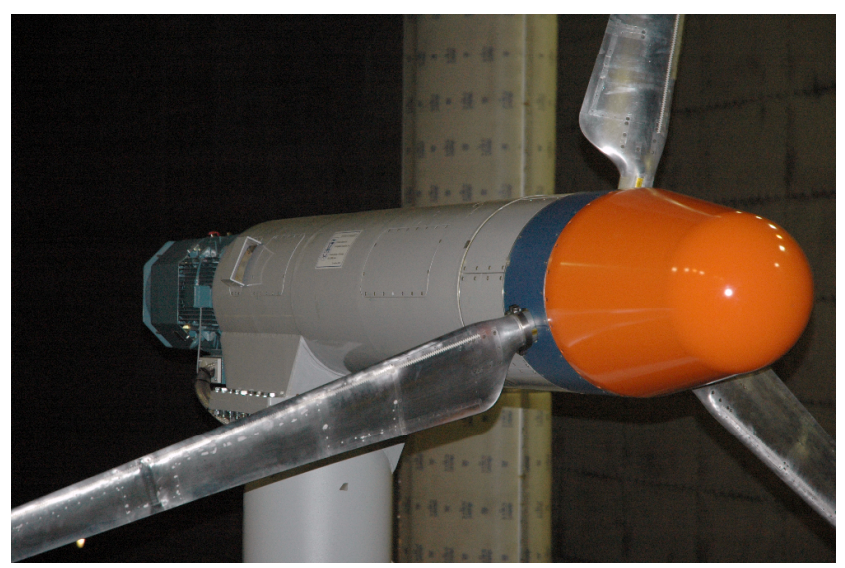

Figure 1: MEXICO rotor used for the experimental campaign.

flow conditions is given. Particle Image Velocimetry (PIV) data in yawed flow is only available for the $\pm 30^{\circ}$ cases with a tunnel wind speed of $15 \mathrm{~m} / \mathrm{s}$ and tip speed ratio (TSR) of 6.68 .

With the inverse free-wake(IFW) method, the loads gathered from the experiment can be used as an input in order to derive the velocity field due to the wake. The model uses 3D airfoil data as input. On the other hand, the direct free-wake(DFW) method uses 2D airfoil data as input. A detailed analysis on the sensitivity of the solution to the airfoil data input is performed. First, the differences between the wake geometry due to both models are studied. Furthermore, an investigation on the differences between various flow conditions and loading on the rotor is done.

The major aim of this work is therefore to understand the importance of having 3D airfoil data in a free-wake methodology by means of a validation of models with the MEXICO experimental results.

\section{Models Used in the Analysis}

The BEM model for yawed conditions used in this work is based on that proposed in in [5]. Tip/root loss, wake expansion and wake rotation corrections are employed. The Glauert correction due to the turbulent wake state in yawed flow is not implemented. The reader is referred to [4] for more details on the BEM model.

The lifting-line, inverse free-wake model methodology is shown in figure 2 . The experimental loads are used as input for the model, and assuming an angle of attack distribution, the velocity field solution is obtained. The new calculated angle of attack is used for the next iteration until a suitable convergence criterion is satisfied as explained in [6]. A number of corrections have been employed in the simulations including vortex filament stretching, Lamb-Oseen vortex core as well as core expansion model [7]. Various verification tests have been performed to ensure a good grid independence of the results. Table 1 shows the wake discretization parameters under the IFW coloumn. The verification tests led to errors in bound circulation lower than $1.5 \%$ for the discretization used in these cases.

The direct free-wake model also uses a lifting line approach. The methodology is shown in figure 3. The reader is referred to [10] for further information on the model. 


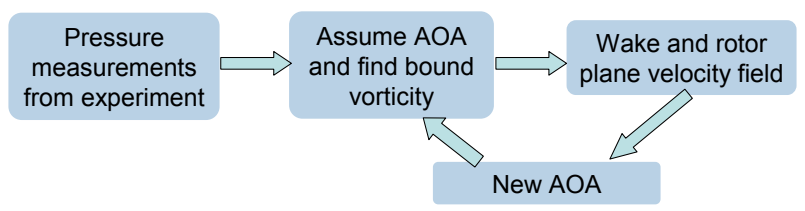

Figure 2: Inverse free wake methodology.

The same corrections as in the inverse free-wake approach are used. Grid independence tests have been also carried out. The wake discretization parameters are shown in table 1 under the DFW coloumn.

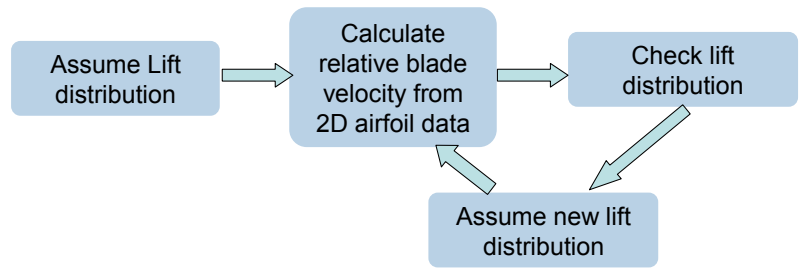

Figure 3: Direct free wake methodology.

\begin{tabular}{rrr} 
& IFW & DFW \\
\hline Blade elements $(N)$ & 21 & 18 \\
Azimuthal Steps per revolution $\left(N_{\phi}\right)$ & 36 & 36 \\
Number of revolutions $\left(N_{\text {rev }}\right)$ & 4 & 4
\end{tabular}

Table 1: Wake discretization parameters to ensure grid independence.

\section{Wake Geometry Predictions}

The PIV results were used to obtain the vorticity for each measurement window downstream in the wake. The position of the maximum vorticity is considered to be the position of the vortex core. This is shown in figure 4. The tip vortex is observed to grow with increasing vortex age. The vorticity is also seen to decrease with wake age due to the diffusion of the vortex. The $\pm 30^{\circ}$ PIV measurements can be combined to give the positions of the tip vortices on a horizontal plane in the wake.

These results can be compared with the IFW and DFW results. With such models, due to the roll up of the trailing vortices, it is difficult to determine the centre of a single tip vortex. Figure 5 shows the results from the IFW simulation. There is a very good agreement of the core positions at the $90^{\circ}$ azimuthal position. At the $270^{\circ}$ position, the agreement is also relatively good but with increasing wake age, the positions of the tip vortices as established from PIV measurements seem to be slighltly more upward than the positions predicted by the IFW model. Figure 6 shows the wake geometry prediction as obtained from the DFW simulation. In this case there is an appreciable difference in the locations of the vortex cores as obtained from the PIV measurements. The tip vortices from the simultation travel faster downstream than the tip vortices observed experimentally. The reason for this has its roots in what is happening at the rotor plane. 


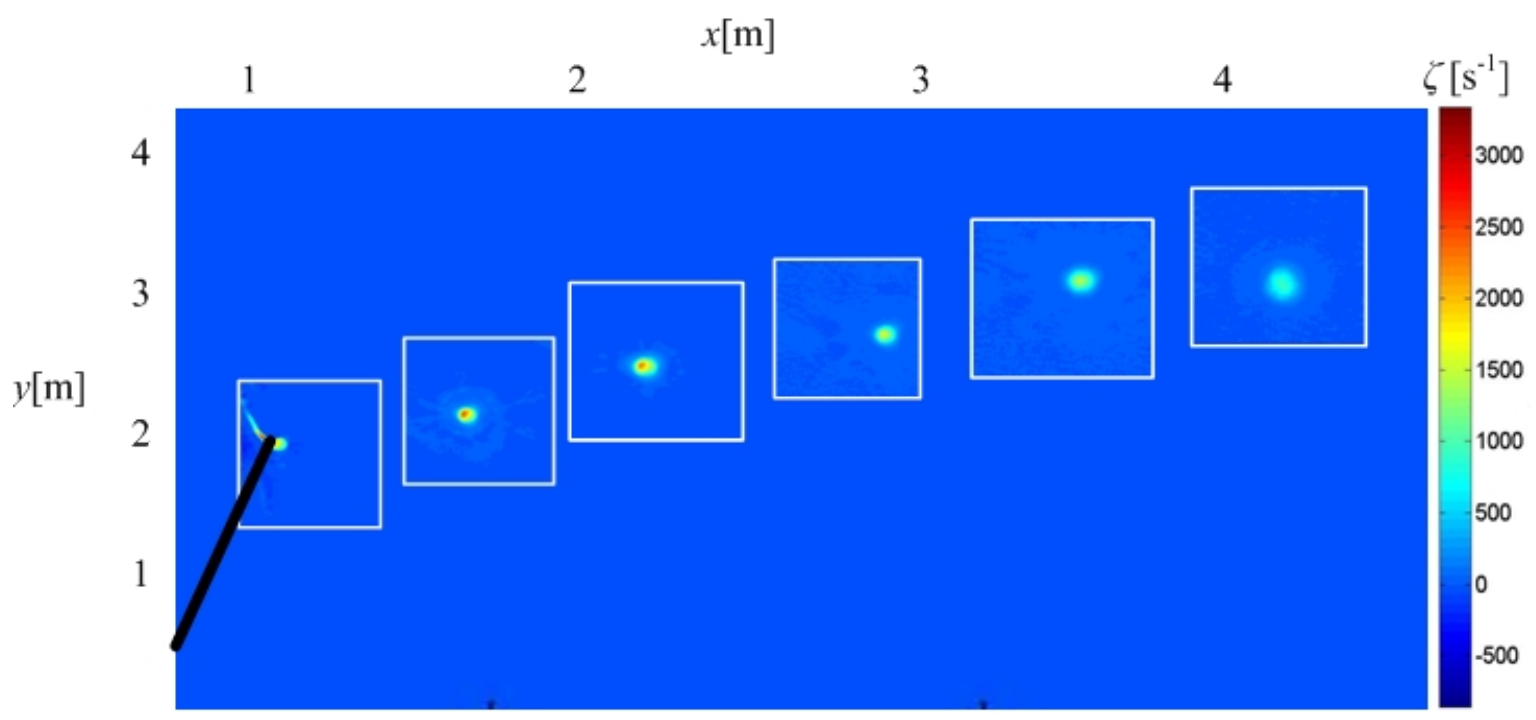

Figure 4: Tip vortex evolution. Vorticity is shown on the contour plot which is obtained from the PIV measurements.

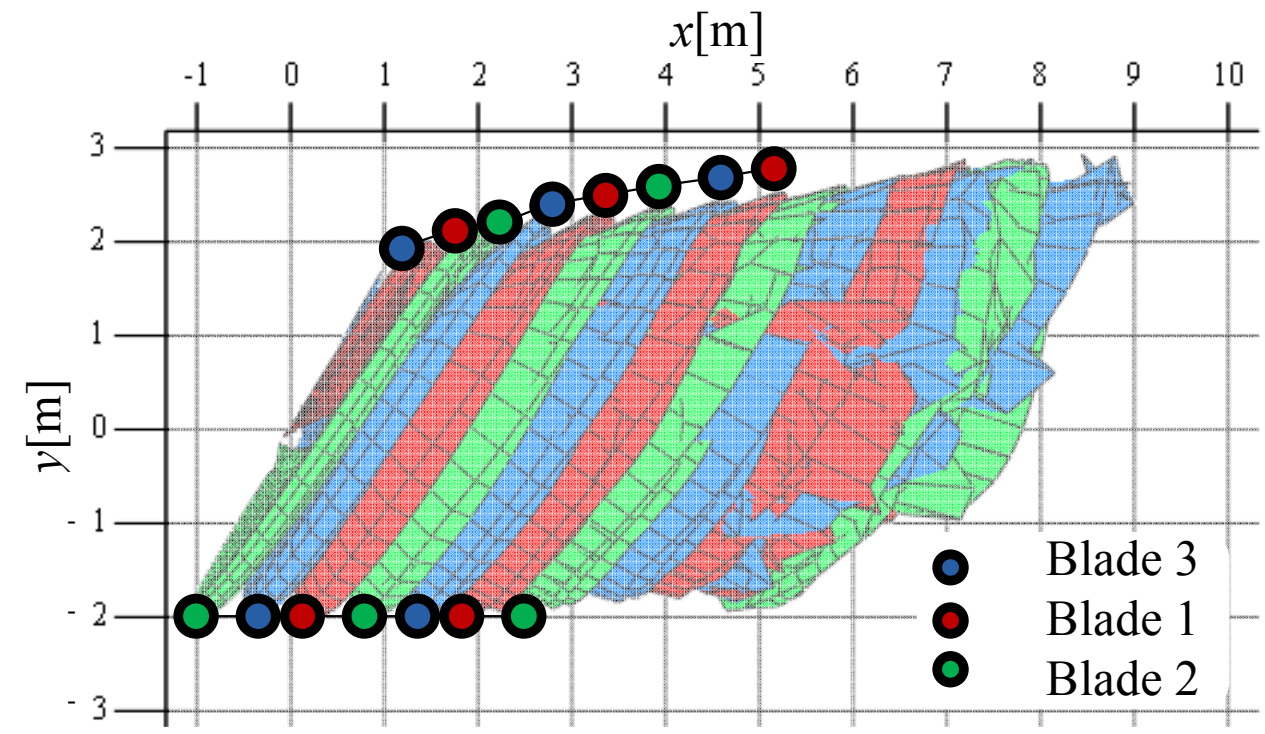

Figure 5: Wake geometry comparison between the inverse free-wake model results and PIV measurements. Yaw $=30^{\circ}, \lambda=6.68$. 


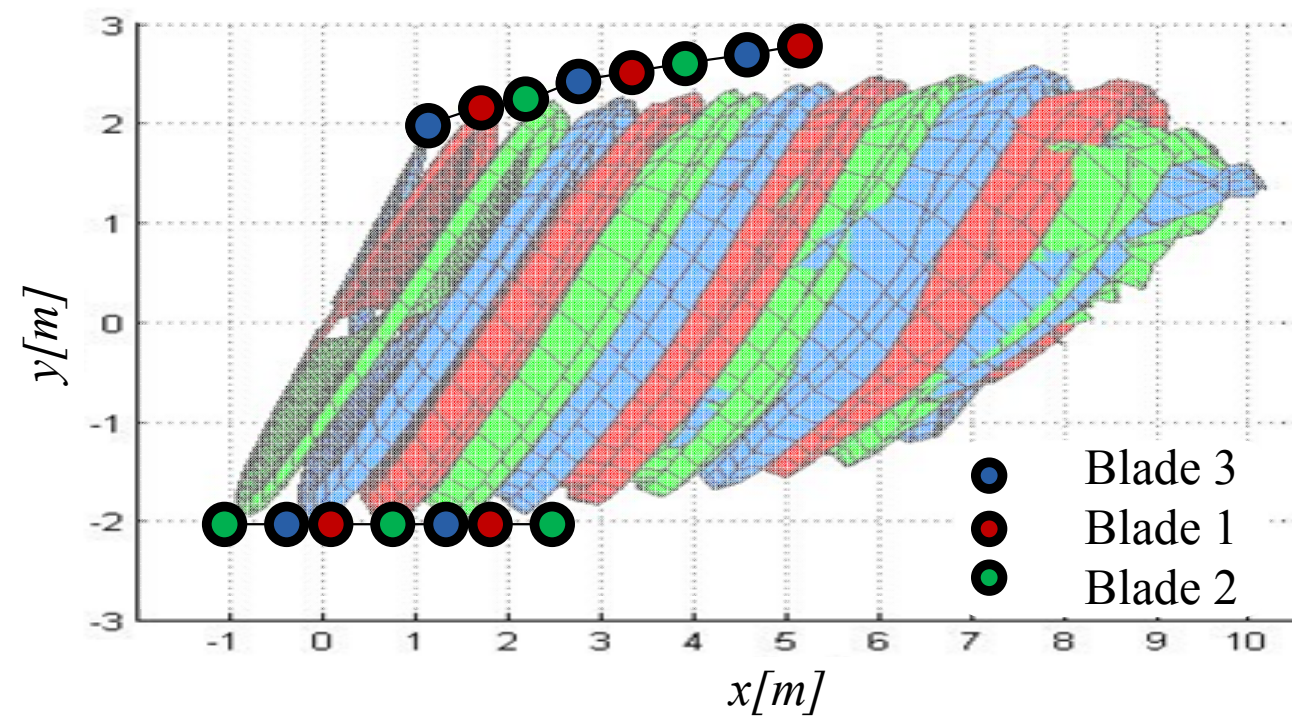

Figure 6: Wake geometry comparison between the direct free-wake model results and PIV measurements. Yaw $=30^{\circ}, \lambda=6.68$.

\section{Conditions at the Rotor Plane}

The derived angle of attack for all three methods is highly dependent on the type of airfoil data which is used. For both BEM and DFW models, 2D steady airfoil data is used. On the other hand, the IFW model makes use of experimental load data which includes 3D effects and unsteadiness. The three results are compared for the entire rotor plane by means of contour plots in figure 8 . There is quite a good agreement between the result from BEM and that from the IFW result. However when the axial blade induction factors are considered, a large disagreement can be observed as shown in figure 7 . The agreement in inductions between the DFW and IFW is much better over an entire blade revolution except at the tips. A difference in inductions is also apparent in the region of the rotor from around $0^{\circ}$ to $180^{\circ}$. The DFW model shows a lower induction in this region due to the faster downstream convection of the trailing vortices.

Hence, the agreement in angle of attack is suspected to occur due to errors in azimuthal induction cancelling with the errors in the axial induction. On the other hand, the differences in angle of attack between the DFW and IFW results is quite large. The differences between the the DFW and IFW angle of attack is shown in figure 9, where a maximum difference of around 4.5 degrees can be observed towards the inboard stations around the 0 degrees azimuthal position.

This difference in angle of attack causes a high difference between the lift coefficients obtained. Lift coefficient against angle of attack for the $92 \%$ span position is shown in figure 10. Towards the tip region, the reduced frequency is low and the flow can be assumed to be steady. Tip effects cause a reduction in lift as was also observed in the NREL Phase VI experiment as shown in [8] and [9]. This can also be observed with the IFW result. Thus, not only is there a difference in the resulting angle of attack due to the 2D data, which is input in a DFW model, but also, the 3D airfoil performance towards 


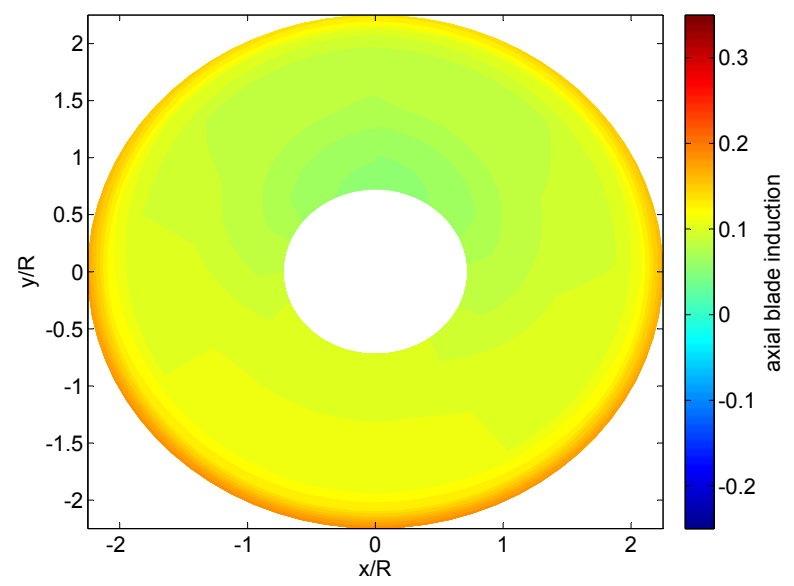

(a)

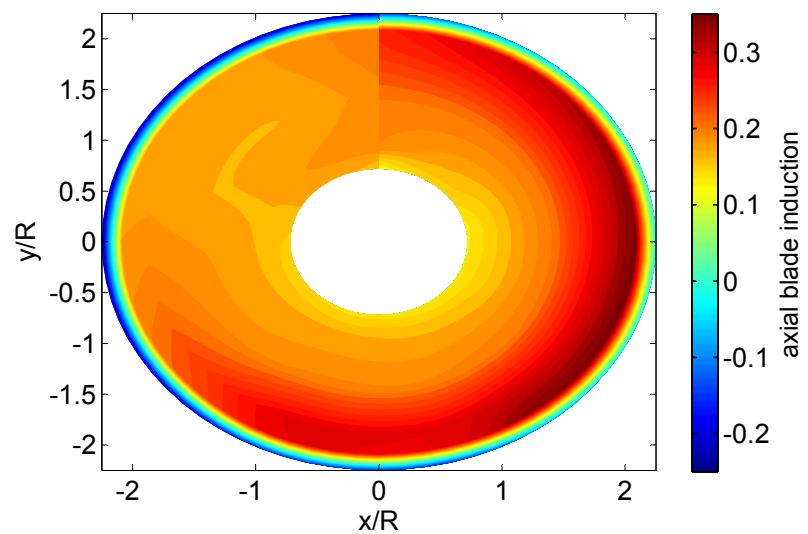

(b)

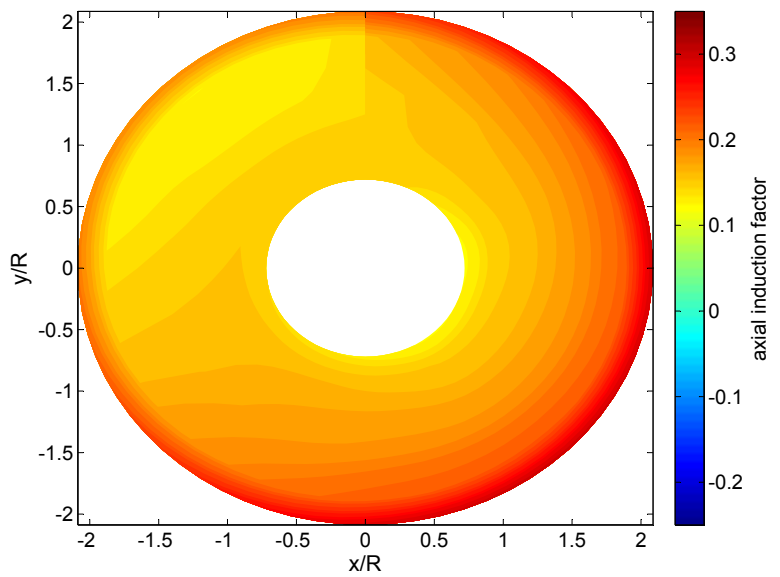

(c)

Figure 7: Blade axial induction contours at the rotor plane as calculated from (a) BEM, (b) IFW and (c) DFW 


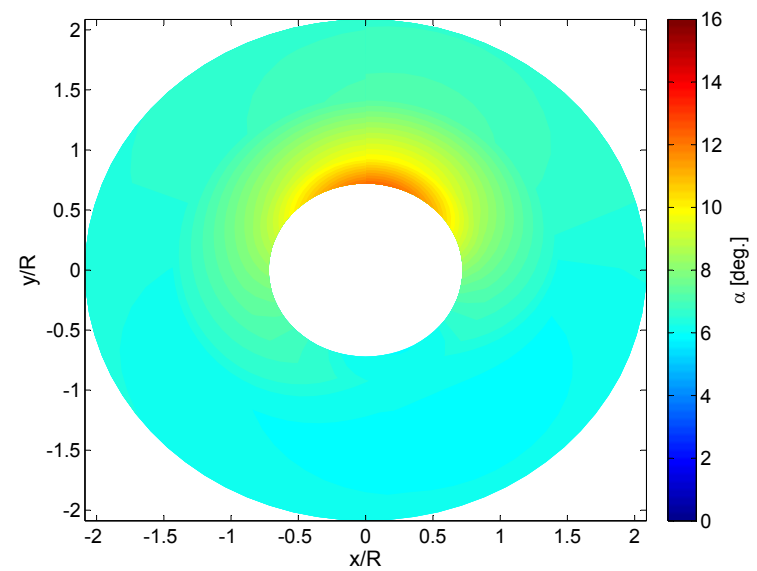

(a)

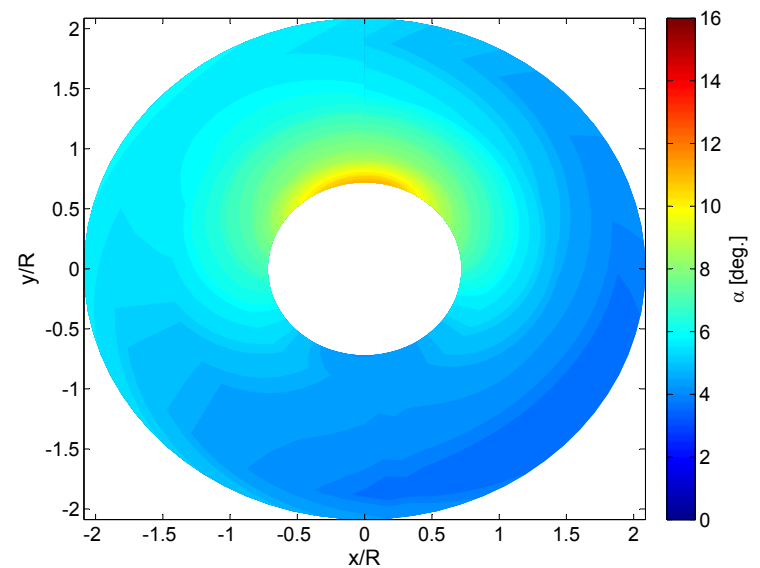

(b)

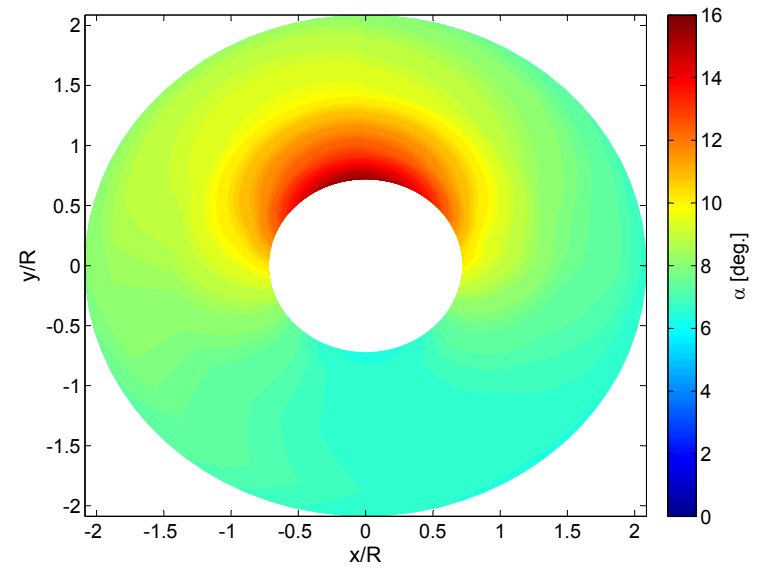

(c)

Figure 8: Angle of attack contours at the rotor plane as calculated from (a) BEM, (b) IFW and (c) DFW 


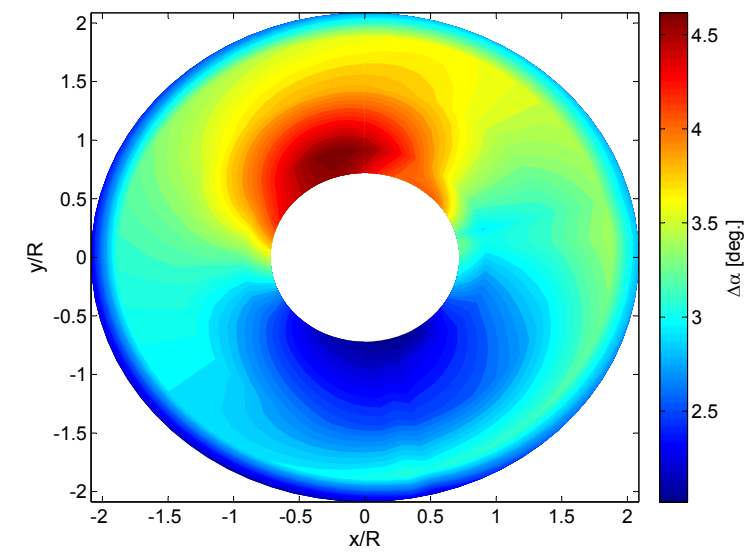

Figure 9: Angle of attack differences between DFW and IFW results.

the tip shows a reduced lift. This causes quite a large difference in the loading of the rotor which is predicted by these models.

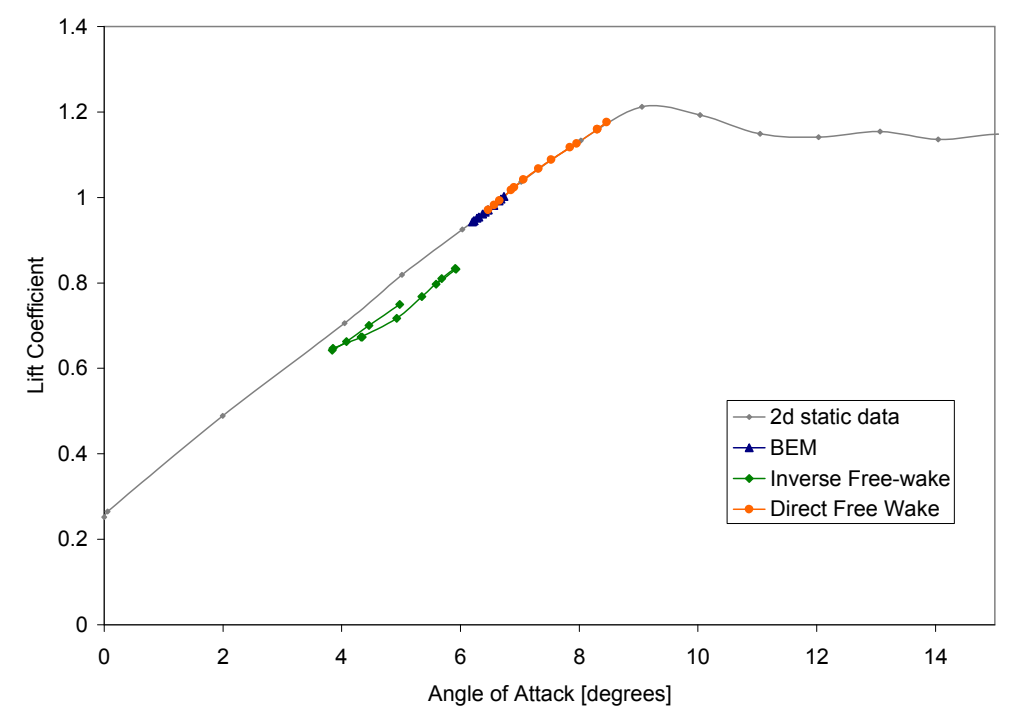

Figure 10: $C_{L}-\alpha$ data for $\beta=30^{\circ}, U_{\infty}=14.97 \mathrm{~m} / \mathrm{s}$, TSR $=6.68$, Span $=92 \%$

As an explanation to the wake geometry differences found with DFW and IFW simulations the difference in lift causes a proportional difference in bound circulation on the blades. This large bound circulation enhances the downwind convection of the vortices. The trailing circulation with the IFW model is found to be slightly higher than that found by means of the DFW model which means that the difference in bound circulation between two adjacent horse-shoe vortices is indeed larger than that predicted with 2D airfoil data. A close up of the near wake is shown in figures 11 and 12 . The effect of this lower trailing vortex circulation is to hinder the wake to skew as it should and as was observed with the PIV measurements (see figure 6). 


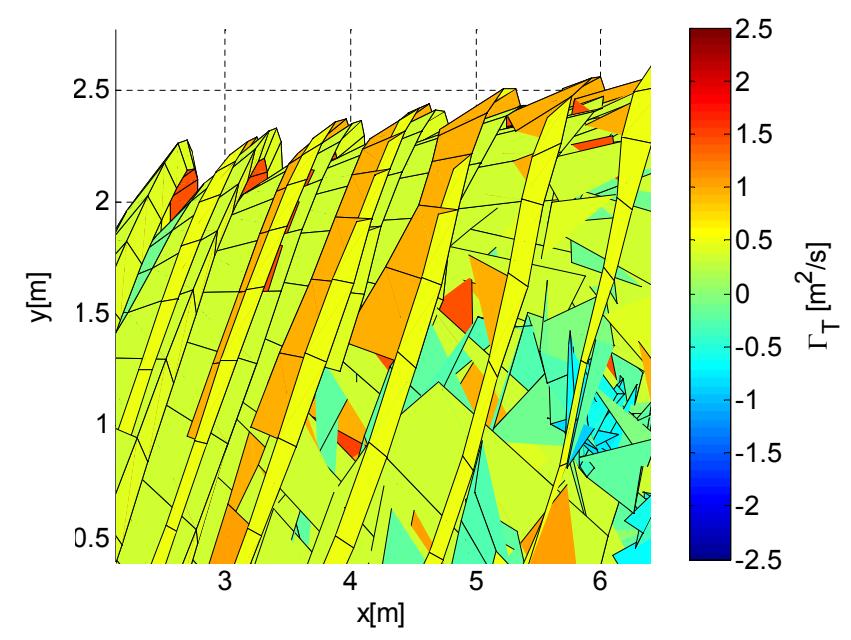

Figure 11: Trailing circulation obtained with the IFW model. Yaw $=30^{\circ}, \lambda=6.68$.

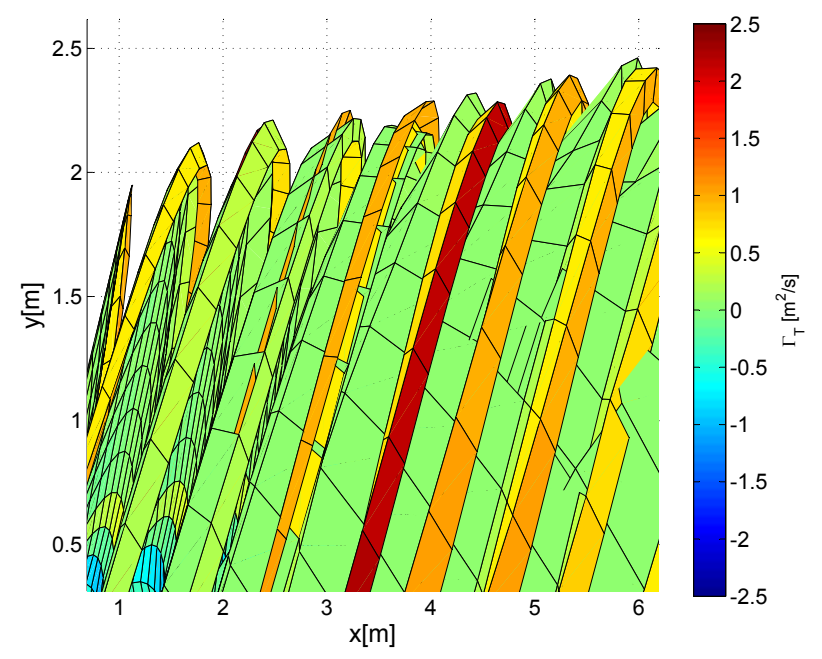

Figure 12: Trailing circulation obtained with the DFW model. Yaw $=30^{\circ}, \lambda=6.68$.

\section{Conclusions}

In this work three models are used to simulate the MEXICO experiment in conditions of yawed flow of $\pm 30^{\circ}$; the simulation results are validated with experimental load measurements and with PIV measurements for tip vortex tracking. Direct free-wake and inverse free-wake are used to predict tip vortex locations for yawed flow conditions. The difference between these two models is due to the prescribed airfoil data. In both BEM and the DFW model, the input is the 2D airfoil data. In the IFW model the input is experimental 3D data.

The results of the IFW model are in better agreement with the experimental results for the convection of the tip vortex, when compared with the results from the DFW model. This difference is due to the airfoil data input. The wake geometry is therefore quite sensitive to the 3D nature of the flow present at the blades. The IFW and DFW showed 
a difference between angles of attack of a maximum of $4.5^{\circ}$. The resulting lift difference was therefore not only attributable to the drop in lift occuring due to the tip vortex but also due to the difference in calculated angles of attack, causing quite a high difference in lift coefficient. The BEM model showed good agreement in angle of attack but when axial induction factors were compared, BEM failed.

The differences in loading conditions at the rotor turn out to affect the shape of the wake which results due to differences in trailing circulation of the vortex filaments used in both IFW and DFW models. From this validation study it may therefore be concluded that in conditions of yawed flow, the resulting load and wake geometry predictions are quite sensitive to the type of airfoil input which is employed. BEM proved unsuitable in predicting the induction at the rotor plane.

\section{References}

[1] Model Experiments in Controlled Conditions(MEXICO) - Final Report

[2] K. Boorsma, J.G. Schepers Description of experimental setup MEXICO measurements, ECN-X-09-0XX.

[3] L. Pascal, Analysis of Mexico measurements- ECN Wind Memo-09-010

[4] D. Micallef, M. Kloosterman, C.S. Ferreira, T. Sant, G. van Bussel, Comparison and Validation of BEM and Free Wake Unsteady Panel Model with the MEXICO Rotor Experiment. Proceedings of Euromech 508 Synposium held at the Universidad Politcnica de Madrid 20-22 October, 2009.

[5] T. Burton, D. Sharpe, N.Jenkins, E. Bossanyi Wind Energy Handbook, Wiley, 2001.

[6] T. Sant, Improving BEM based Aerodynamic Models in Wind Turbine Design Codes - PhD Dissertation, TUDelft/University of Malta

[7] D. Micallef: MEXICO Data Analysis, Stage V - Investigation of the Limitations of Inverse Free Wake Vortex Codes on the Basis of the MEXICO Experiment, ECN.

[8] T. Sant, G. van Kuik, G.J.W. van Bussel, Estimating the Angle of Attack from Blade Pressure Measurements on the National Renewable Energy Laboratory Phase VI Rotor Using a Free Wake Vortex Model: Axial Conditions, Wind Energy, 2006. DOI 10.1002/we.201. pgs. 549-557.

[9] T. Sant, G. van Kuik, G.J.W. van Bussel, Estimating the Angle of Attack from Blade Pressure Measurements on the National Renewable Energy Laboratory Phase VI Rotor Using a Free Wake Vortex Model: Yawed Conditions, Wind Energy, 2009. DOI 10.1002/we.280. pgs. 1-32

[10] M.H.M. Kloosterman, Development of the Near Wake behind a Horizontal Axis Wind Turbine Including the development of a Free Wake Lifting Line Code - M.Sc Dissertation, TUDelft.

[11] J.G. Leishman, Principles of Helicopter Aerodynamics, Cambridge University Press, 2008. 